\title{
Red Nacional de Gestión de Conocimiento, Investigación e Innovación en Tuberculosis en Colombia
}

\author{
Carlos Eduardo Rincón-Torres ${ }^{1}$, Vivian Rubio², Claudia Castro ${ }^{3}$, Ingrid García ${ }^{4}$, Oscar Andrés Cruz ${ }^{5}$, \\ Julián Trujillo-Trujillo ${ }^{5}$ y Gloria Mercedes Puerto ${ }^{2}$
}

Forma de citar Rincón-Torres CE, Rubio V, Castro C, García I, Cruz OA, Trujillo-Trujillo J, et al. Red Nacional de Gestión de Conocimiento, Investigación e Innovación en tuberculosis en Colombia. Rev Panam Salud Publica. 2021;45:e23. https://doi.org/10.26633/RPSP.2021.23

RESUMEN El Ministerio de Salud y Protección Social de Colombia, en colaboración con el Instituto Nacional de Salud y
la Organización Panamericana de la Salud, convocaron en 2018 a todos los actores asociados al Programa
Nacional de Prevención y Control de la Tuberculosis (PNPCT) y el Sistema Nacional de Ciencia, Tecnología e
Innovación a la conformación de una red nacional que coordinara la investigación operativa (IO) relacionada
con la tuberculosis (TB) en el país. Se realizaron siete talleres en los que se llegó al consenso de establecer
la Red Nacional de Gestión de Conocimiento, Investigación e Innovación en Tuberculosis en Colombia (Red
TB Colombia). Con más de 180 participantes, organizados en un nodo central y cinco nodos regionales que
agrupan a las 37 entidades territoriales, en 2019 la Red TB Colombia realizó cuatro jornadas de capacitación
en IO y definió 65 temas priorizados de investigación, ajustados a las líneas estratégicas establecidas por
el PNPCT: prevención y atención integral, con foco en los afectados por la TB (41 temas en tres módulos) y
compromiso político, protección social y sistemas de apoyo (24 temas en cuatro módulos). La priorización se
realizó por el método de comparación por pares. La Red TB Colombia sigue activa y contribuye a la gestión
del conocimiento dirigido a eliminar la TB en el país y ha permitido avanzar en la construcción de planes y
la ejecución de proyectos locales de IO que generan resultados científicamente validados para fortalecer el
PNPCT y mejorar la atención de los pacientes con TB.

Palabras clave Tuberculosis; programas nacionales de salud; desarrollo sostenible; Colombia.

La tuberculosis (TB) sigue siendo una amenaza para la salud pública en el mundo con 10 millones de casos registrados en 2019 (1). Ese año, en Colombia se registraron 14886 casos, para una incidencia de 27,3 por 100000 habitantes (2).

En 2014 y 2015, los países miembros de la Organización Mundial de la Salud (OMS) —incluida Colombia - se comprometieron a eliminar la epidemia de TB mediante la adopción de la Estrategia Fin a la TB, de la OMS (3), y los Objetivos de Desarrollo Sostenible, de las Naciones Unidas (4).

En Colombia, el Programa Nacional de Prevención y Control de la Tuberculosis (PNPCT), liderado por el Ministerio de Salud y Protección Social (MSPS), adoptó la estrategia de la OMS (5)

\footnotetext{
Subdirección de Innovación en Salud Pública, Instituto Nacional de Salud, Bogotá, Colombia.

2 Grupo de Micobacterias, Subdirección de Investigación Científica y Tecnológica, Instituto Nacional de Salud, Bogotá, Colombia. $\bowtie$ Gloria Mercedes Puerto, gpuerto@ins.gov.co

3 Equipo Banco de Proyectos, Dirección de Investigación en Salud Pública, Instituto Nacional de Salud, Bogotá, Colombia.
}

\footnotetext{
4 Unidad de Control de Enfermedades, Organización Panamericana de la Salud, Bogotá, Colombia.

5 Programa Nacional de Prevención y Control de la Tuberculosis, Dirección de Promoción y Prevención, Ministerio de Salud y Protección Social, Bogotá, Colombia.
} 
mediante el Plan Estratégico Hacia el Fin de la TB 2016-2035 (6), basado en tres líneas estratégicas:

1) prevención y atención integral, con foco en los afectados por la TB

2) compromiso político, protección social y sistemas de apoyo

3) investigación operativa (IO) e implementación de iniciativas y herramientas innovadoras para el control de la TB.

Para dar respuesta a esta última línea estratégica, se creó la Red Nacional de Gestión de Conocimiento, Investigación e Innovación en Tuberculosis en Colombia (Red TB Colombia), en la que se desarrolla e implementa un plan nacional de investigación para la generación de nuevas evidencias científicas que contribuyan al cumplimiento de las acciones del PNPCT (6).

En el presente trabajo se describen los antecedentes, las características, los logros y las perspectivas de la Red TB Colombia.

\section{ANTECEDENTES}

A partir de lo concertado en la Primera Conferencia Ministerial de la OMS "Poner fin a la tuberculosis en la era del desarrollo sostenible: una respuesta multisectorial", celebrada en 2017 en Moscú, Rusia (7), y la Reunión de Alto Nivel de la Asamblea General de las Naciones Unidas Sobre el Fin de la Tuberculosis, llevada a cabo en 2018 en Ginebra, Suiza, se insistió en la necesidad de impulsar la ciencia, las investigaciones y los mecanismos de colaboración entre países para alcanzar ese objetivo común (8).

Bajo ese marco de acción, en América Latina se han creado redes de investigación dedicadas a la TB; Brasil fue el país pionero con la creación en el 2001 de la REDE-TB (9), que en 2018 ya contaba con 320 miembros de 65 instituciones que participaron en la consolidación de la Agenda Nacional de Investigación en Tuberculosis de ese país (10).

En Perú se creó la Red Nacional de Investigación en Tuberculosis en 2017, bajo la dirección del Ministerio de Salud y el Instituto Nacional de Salud de ese país e integrada por investigadores e instituciones, tanto públicas como privadas. Unos años antes, en el país se había establecido la Agenda Nacional de Investigación en Tuberculosis 2011-2014, en la que participaban investigadores, coordinadores regionales del programa de TB y académicos $(11,12)$.

\section{Constitución de la Red TB Colombia}

Para dar respuesta a lo establecido en la política nacional de lucha contra la TB, el MSPS — como entidad rectora del sistema de salud - en colaboración con el Instituto Nacional de Salud (INS) y la Oficina de la Organización Panamericana de la Salud (OPS) y la OMS en Colombia, convocaron en 2018 a la conformación de una red nacional con el fin de promover la IO relacionada con la TB en el país. Se invitó a participar a todos los actores involucrados en el trabajo del PNPCT y el Sistema Nacional de Ciencia, Tecnología e Innovación, incluidos las entidades territoriales (departamentos, distritos, municipios y territorios indígenas) y administradoras de planes de beneficios, los representantes de la sociedad civil, las entidades de salud pública, y los investigadores y académicos vinculados a la TB. Se definió como operativa, la investigación dirigida a mejorar la operación del programa y brindar una atención más eficaz, eficiente y equitativa, mediante la identificación de los problemas - a partir de los recursos y los datos existentes recopilados habitualmente por el PNPCT - y la orientación del trabajo de obtención de los conocimientos necesarios (13).

Para ello se realizaron siete talleres en los que más de 180 profesionales analizaron los procesos de ideación, planificación e implementación de las estructuras organizacionales de la red. El procedimiento de consenso implicó la concertación, el diálogo y la orientación de decisiones, acciones y recursos individuales dirigidos a objetivos comunes. Como resultado, se decidió establecer la Red TB Colombia, que se basaría en cinco principios básicos:

a) Sería una red formal, definida como una estructura organizacional en red con un gobierno corporativo capaz de tomar decisiones; la Red estaría subordinada al PNPCT y su coordinación estaría a cargo de la Dirección de Investigación en Salud Pública del INS (14).

b) Sería una red permanente, establecida por un tiempo determinado y fijo con horizonte al 2035.

c) Sería una red no similar, integrada y externa, es decir, compuesta por actores con conocimientos y experiencia práctica heterogéneos pertenecientes a entidades e instituciones diversas de carácter tanto público como privado.

d) La red podría recibir financiación de cooperantes privados y del Estado.

e) La red tendría un alto grado de integración y operaría mediante el Nodo Nacional Central (constituido por el MSPS, el INS y la OPS), encargado del acompañamiento técnico a las entidades territoriales organizadas en cinco nodos regionales (Amazonía y Orinoquía, Andino, Atlántico, Pacífico y Eje Cafetero), que agrupan a las 37 entidades territoriales.

Como misión, la red definió "somos un conjunto de actores y sectores público-privados, que de forma organizada, articulada y voluntaria se unen para gestionar conocimiento, investigación e innovación en tuberculosis, con el fin de orientar la toma de decisiones basadas en evidencia que contribuya al control de la enfermedad y su eliminación en Colombia", entendiendo como eliminación la incidencia anual de menos de 1 caso de TB por millón de habitantes. Eso se complementó con la visión de "ser para 2025 un referente nacional en gestión del conocimiento, investigación e innovación en tuberculosis, con sostenibilidad financiera y operativa e incidencia en políticas públicas".

Se adoptó también el principio ético de equidad y se asumieron como valores la excelencia, la confianza y la transparencia financiera y operativa, entre otros. Finalmente, se establecieron indicadores de proceso y de impacto para la evaluación prospectiva a cargo del Nodo Nacional Central (cuadro 1).

La pertenencia a la red por parte de las instituciones y los actores se formaliza mediante cartas de compromiso y cooperación presentadas al INS.

\section{LOGROS EN EL 2020}

A través de la Red TB Colombia, y con el acompañamiento de la academia, se diseñó un taller teórico-práctico en el que los integrantes de los cinco nodos regionales se capacitaron 
CUADRO 1. Indicadores de proceso y de impacto definidos para la evaluación prospectiva de la Red TB Colombia

\begin{tabular}{|c|c|c|c|c|}
\hline Tipo y nombre del indicador & Definición & Medio de verificación & Meta al 2020 & Meta al 2025 \\
\hline \multicolumn{5}{|l|}{ Proceso } \\
\hline $\begin{array}{l}\text { Porcentaje de entidades } \\
\text { territoriales que participan en } \\
\text { la Red TB Colombia }\end{array}$ & $\begin{array}{l}\text { Porcentaje del total de entidades } \\
\text { territoriales que participan } \\
\text { activamente en la red y se reúnen } \\
\text { regularmente }\end{array}$ & $\begin{array}{l}\text { Cartas de compromiso por } \\
\text { entidad territorial recibidas en el } \\
\text { INS como coordinador de la Red }\end{array}$ & $\begin{array}{l}\text { Participación del } 50 \% \text { de las } \\
\text { entidades territoriales (19/37) }\end{array}$ & $\begin{array}{l}\text { Participación del } 80 \% \text { de las } \\
\text { entidades territoriales (30/37) }\end{array}$ \\
\hline $\begin{array}{l}\text { Porcentaje de entidades } \\
\text { territoriales con planes de } \\
\text { investigación en TB }\end{array}$ & $\begin{array}{l}\text { Porcentaje del total de entidades } \\
\text { territoriales, que tienen planes de } \\
\text { investigación en TB, definidos y } \\
\text { con temáticas prioritarias }\end{array}$ & $\begin{array}{l}\text { Planes de investigación en TB } \\
\text { presentados al Nodo Nacional } \\
\text { Central }\end{array}$ & $\begin{array}{l}\text { Definición de planes territoriales } \\
\text { en el } 50 \% \text { de las entidades } \\
\text { territoriales (19/37) }\end{array}$ & $\begin{array}{l}\text { Definición de planes territoriales } \\
\text { en el } 80 \% \text { de las entidades } \\
\text { territoriales (30/37) }\end{array}$ \\
\hline Financiación de la red & $\begin{array}{l}\text { Porcentaje del total de entidades } \\
\text { territoriales, que hacen uso del } \\
\text { mecanismo nacional sostenible } \\
\text { para el financiamiento de la } \\
\text { investigación en TB }\end{array}$ & $\begin{array}{l}\text { Proyectos de investigación } \\
\text { financiados en ejecución }\end{array}$ & $\begin{array}{l}\text { Financiación de proyectos en el } \\
50 \% \text { de las entidades territoriales } \\
\text { que registran el } 50 \% \text { de la carga } \\
\text { de TB en el país }(2 / 4)\end{array}$ & $\begin{array}{l}\text { Financiación de proyectos en el } \\
80 \% \text { de las entidades territoriales } \\
\text { que registran el } 80 \% \text { de la carga } \\
\text { de TB en el país }(8 / 10)\end{array}$ \\
\hline Plan de formación & $\begin{array}{l}\text { Porcentaje del total de entidades } \\
\text { territoriales, que replican el plan } \\
\text { de formación en investigación } \\
\text { operativa en sus territorios }\end{array}$ & $\begin{array}{l}\text { Listas de asistencia a los planes } \\
\text { de formación en las entidades } \\
\text { territoriales }\end{array}$ & $\begin{array}{l}\text { Formación de los recursos } \\
\text { humanos que trabajan en TB } \\
\text { pertenecientes a las entidades } \\
\text { territoriales que registran el } 50 \% \\
\text { de la carga de TB en el país }(2 / 4)\end{array}$ & $\begin{array}{l}\text { Formación de los recursos } \\
\text { humanos que trabajan en } \\
\text { TB pertenecientes a las } \\
\text { entidades territoriales que } \\
\text { registran el } 80 \% \text { de la carga de TB } \\
\text { en el país }(8 / 10)\end{array}$ \\
\hline $\begin{array}{l}\text { Iniciativas y herramientas } \\
\text { innovadoras }\end{array}$ & $\begin{array}{l}\text { Número de iniciativas y } \\
\text { herramientas innovadoras, } \\
\text { multisectoriales e integradas, a } \\
\text { nivel nacional o internacional, } \\
\text { identificadas e incorporadas, para } \\
\text { el mejoramiento de la respuesta } \\
\text { a la TB }\end{array}$ & $\begin{array}{l}\text { Registro de iniciativas ante el } \\
\text { Nodo Nacional Central }\end{array}$ & Al menos una & Más de una \\
\hline \multicolumn{5}{|l|}{ Impacto } \\
\hline $\begin{array}{l}\text { Propuestas para el control del } \\
\text { Programa Nacional de } \\
\text { Prevención y Control de la } \\
\text { Tuberculosis }\end{array}$ & $\begin{array}{l}\text { Número de estrategias para } \\
\text { el control del Programa } \\
\text { propuestas como resultado de } \\
\text { la investigación operativa }\end{array}$ & $\begin{array}{l}\text { Nuevas políticas o estrategias } \\
\text { basadas en estudios de } \\
\text { investigación operativa } \\
\text { presentadas al MSPS }\end{array}$ & NA & Al menos una \\
\hline
\end{tabular}

Fuente: elaborado por los autores.

Nota: INS: Instituto Nacional de Salud; TB: tuberculosis; MSPS: Ministerio de Salud y Protección Social; NA: no aplica.

en la formulación de protocolos y planes de IO, según la Guía para la Implementación de la Estrategia Fin de la TB (15), que contiene los aspectos esenciales recomendados por la OMS.

Con el propósito de conocer los recursos humanos y de infraestructura disponibles en las entidades territoriales y los centros de investigación que conforman la red, se aplicaron dos encuestas. En cuanto a los recursos humanos, los resultados mostraron que el $43 \%$ del talento humano de la red son profesionales especializados, el 36\% cuenta con maestrías, el $14 \%$ con doctorados y el $4 \%$ con posdoctorados; el $3 \%$ restante tiene formación profesional sin especialización. En cuanto a las áreas de experiencia y conocimientos de los profesionales especializados, el $46 \%$ correspondió a salud pública, el $20 \%$ a epidemiología, el 17\% a microbiología, el 5\% a biología molecular y el 12\% restante se distribuía en el área clínica, las ciencias sociales y la gestión de riesgos.

Con respecto a la infraestructura, el $80 \%$ de las instituciones participantes contaba con instalaciones adecuadas para la realización de actividades de investigación; de ellas, el 37\% cumplía con el nivel de bioseguridad I, el 50\% con el nivel de bioseguridad II, el 10\% con el nivel de bioseguridad II plus y el 3\% con el nivel de bioseguridad III.

De los integrantes de la red, actualmente el $48 \%$ dedica al menos 5 horas semanales a investigaciones relacionadas con la
TB y el 28\% dedica a ello más de 10 horas; el 24\% que no tiene establecido tiempo para la investigación manifestó su intención de vincularse a los proyectos de IO.

Para la financiación y el funcionamiento de la Red TB Colombia, en 2019 el MSPS aprobó un fondo "semilla" mediante una iniciativa que destinó hasta el 10\% de los recursos económicos del PNPCT, transferidos por el MSPS a las secretarías de salud de las entidades locales, para la financiación de investigaciones que aportaran al mejoramiento de sus indicadores programáticos o que contribuyeran a orientar mejor la toma de decisiones a nivel operativo (16). Adicionalmente, la Dirección de Investigación en Salud Pública del INS se encargó de identificar y comunicar a los miembros de la Red las oportunidades disponibles de financiación nacional e internacional. Mediante estos mecanismos se promueve la realización de proyectos de IO dirigidos a mejorar los indicadores programáticos y orientar la toma de decisiones a nivel operativo.

En 2019 se establecieron los niveles de prioridad de las temáticas de investigación en TB para todo el país. Mediante la técnica conocida como lluvia o tormenta de ideas se identificaron 65 temas priorizados, basados en el Marco Global de Acción para la Investigación en TB (17), los que se organizaron en módulos según las dos primeras líneas estratégicas nacionales mencionadas más arriba. Se incluyeron 41 temas en la línea estratégica 1 (prevención y atención integral, con foco en los afectados por 
CUADRO 2. Temáticas priorizadas por los integrantes de la Red TB Colombia para la investigación en tuberculosis (TB) en Colombia, 2019

Módulo según la línea estratégica nacional

Línea estratégica 1: Prevención y atención integral, con foco en los afectados por la TB

Detección y diagnóstico de la TB en Detección y diagnóstico de la TB en niños población general y vulnerable

Tratamiento de la TB en población general y vulnerable

Epidemiología

Estudio de contactos

Diagnóstico de la TB y farmacorresistencia en diferentes grupos poblacionales Detección y diagnóstico de la TB en poblaciones vulnerables ${ }^{b}$

Detección y diagnóstico de la TB en pacientes con comorbilidades tales como diabetes, cáncer, inmunodeficiencias, enfermedades renales, enfermedad pulmonar obstructiva crónica y silicosis

Tratamiento y seguimiento a casos de TB pertenecientes a poblaciones vulnerables ${ }^{b}$

Factores culturales y sociales que afectan a la adhesión al tratamiento

Uso de nuevas tecnologías de la información y las telecomunicaciones, como la telemedicina y las plataformas virtuales, para la supervisión del tratamiento, el seguimiento y el apoyo psicosocial

Farmacogenómica aplicada a mejorar la respuesta al tratamiento de fármacos antituberculosos

Factores relacionados con el éxito del tratamiento

Factores relacionados con la adhesión al tratamiento

Implementación de sistemas de monitoreo epidemiológicos de la TB

Línea estratégica 2: Compromiso político, protección social y sistemas de apoyo

Compromiso político, protección social y sistemas de apoyo

Abordaje de las inequidades sociales relacionadas con la TB

Acción participativa para aumentar las capacidades en salud pública en regiones y municipios

Proyectos que incluyan la protección social al paciente con TB y su familia

Costo catastrófico por TB

Sistema de salud

Estudio de barreras administrativas desde el sistema de salud y su superación

Evaluación integral (diseño, focalización, cobertura, implementación, procesos, resultados, impacto y aspectos económicos) del PNPCT

Prestación de servicios de salud, cobertura universal de salud y atención integral

Sistema nacional de información del diagnóstico y el tratamiento de los pacientes con TB

Sistemas de información Implementación de programas informáticos para la captura y el análisis de los datos del PNPCT Historia clínica del paciente en línea en cualquier lugar del país y en tiempo real

Articulación de los datos de Sivigila y el PNPCT en una base nacional única

Sistematización de las experiencias de investigación

Sistemas de control de infecciones

Tuberculosis ocupacional

Medidas de control ambiental

Medidas de protección respiratoria

Medidas de control administrativo

Fuente: elaborado por los autores.

Nota: PNPCT: Programa Nacional de Prevención y Control de la Tuberculosis; Sivigila: Sistema de Vigilancia en Salud Pública.

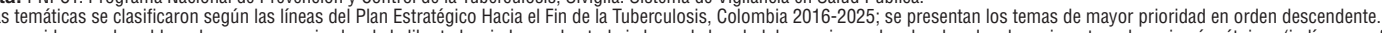

${ }^{b}$ Se consideran vulnerables a las personas privadas de la libertad y sin hogar, los trabajadores de la salud, los ancianos, los desplazados, los migrantes y las minorías étnicas (indígenas, afrodescendientes, romaníes o gitanos, y raizales).

la TB) agrupados en tres módulos: detección y diagnóstico de la TB en población general y vulnerable; tratamiento de la TB en población general y vulnerable; y epidemiología; se incluyeron 24 temas en la línea estratégica 2 (compromiso político, protección social y sistemas de apoyo) en cuatro módulos: compromiso político, protección social y sistemas de apoyo; sistema de salud; sistemas de información; y sistemas de control de infecciones (cuadro 2). La priorización se realizó por el método de comparación por pares (18).

\section{PERSPECTIVA COLOMBIANA}

Antes de la creación de la Red TB Colombia, en el país se realizaba la investigación relacionada con la TB principalmente en centros de investigación y académicos de tres ciudades: Bogotá, Medellín y Cali. Un importante ejemplo es el Centro Colombiano de Investigación en Tuberculosis, que operó entre el 2005 y el 2012 con financiación de Colciencias y que realizó un trabajo articulado que aportó resultados relevantes para el PNPCT (19-21). Con la constitución de la Red TB Colombia se espera que la investigación continúe extendiéndose a todo el territorio nacional y llegue a todas las poblaciones de Colombia para dar solución a los problemas de la TB en todo el país; se espera mejorar la capacidad de los recursos humanos mediante la transferencia de conocimientos entre profesionales de las diferentes entidades territoriales.

Como principales retos para el año 2021 se prevé diseñar un curso teórico-práctico en línea para capacitar un mayor número de actores, y acompañar a todas las entidades territoriales en la formulación de planes y proyectos regionales en IO.

La principal limitación para el buen desempeño de la red es la escasez de recursos financieros para desarrollar más investigaciones locales en los propios territorios. 


\section{CONCLUSIONES Y RECOMENDACIONES}

La Red TB Colombia está activa y contribuye a la gestión del conocimiento dirigido a poner fin a la TB en el país. A partir de su Nodo Nacional Central y sus cinco nodos regionales, esta red ha permitido avanzar en la construcción de planes y en la ejecución de proyectos locales de IO encaminados a generar resultados científicamente validados para fortalecer el PNPCT y mejorar la atención de los pacientes con TB. Se espera continuar la consolidación de la Red de manera que sus resultados incidan positivamente en la generación de nuevas políticas y estrategias que fortalezcan la lucha contra este flagelo.

Las redes de investigación en TB deben articular a todos los actores interesados en unir esfuerzos, y recursos humanos y financieros para poner fin a la TB. Estas redes deben incluir a entidades gubernamentales, académicas, científicas y representantes de la sociedad civil, bajo la coordinación de los programas de control y las entidades nacionales rectoras de la salud pública, que deben facilitar la convocatoria de los actores y generar compromisos. La construcción participativa de la red desde su inicio es fundamental para fomentar el sentido de pertinencia de sus integrantes.
Contribución de los autores. Todos los autores contribuyeron en la recolección de los datos, el análisis y la interpretación de los resultados, y la escritura y la revisión del manuscrito. CERT, IG, OAC, JTT y GMP participaron en el diseño del estudio. Todos los autores revisaron y aprobaron la versión final.

Agradecimientos. A todas las personas y entidades que conforman y han hecho posible la constitución y puesta en marcha de la Red TB Colombia.

\section{Conflictos de intereses. Ninguno.}

Financiación. Este trabajo contó con el apoyo financiero del INS, el MSPS y la Oficina de la OPS/OMS en Colombia

Declaración. Las opiniones expresadas en este artículo son responsabilidad de los autores y no reflejan necesariamente los criterios ni la política de la Revista Panamericana de Salud Pública / Pan American Journal of Public Health y/o de la Organización Panamericana de la Salud.

\section{REFERENCIAS}

1. World Health Organization. Global tuberculosis report 2020. Geneva: WHO; 2020.

2. República de Colombia, Ministerio de Salud y Protección Social. Programa Nacional de Prevención y Control de la Tuberculosis - PNPCT. Situación de la TB en Colombia: avances en el Plan Estratégico Nacional. Bogotá: MSPS; 2020. Disponible en https://www. minsalud.gov.co/salud/publica/PET/Paginas/Tuberculosis.aspx

3. Uplekar M, Weil D, Lonnroth K, Jaramillo E, Lienhardt C, Dias HM, et al. WHO's new End TB Strategy. Lancet. 2015;385(9979):1799-801 [consultado el 12 de marzo del 2020]. Disponible en: https://doi. org/10.1016/S0140-6736(15)60570-0

4. Naciones Unidas. La Agenda 2030 y los Objetivos de Desarrollo Sostenible: una oportunidad para América Latina y el Caribe. Santiago: NU; 2018. (LC/G.2681-P/Rev.3).

5. Organización Mundial de la Salud. Estrategia Mundial Fin a la Tuberculosis 2016-2035. Ginebra: OMS; 2015 [consultado el 24 de marzo del 2020]. Disponible en: https://www.who.int/tb/strategy /end-tb/es/

6. República de Colombia, Ministerio de Salud y Protección Social. Plan Estratégico Hacia el Fin de la Tuberculosis. Colombia 20162025. Bogotá: MSPS; 2016 [consultado el 24 de marzo del 2020]. Disponible en: https://www.minsalud.gov.co/sites/rid/Lists/Bib liotecaDigital/RIDE/INEC/INTOR/Plan-estrategico-fin-tubercu losis-colombia-2016-2025.pdf

7. Organización Mundial de la Salud. Primera Conferencia Ministerial Mundial de la OMS. Poner fin a la tuberculosis en la era del desarrollo sostenible: una respuesta multisectorial. Moscú: OMS; 2017 [consultado el 24 de marzo del 2020]. Disponible en: https: / / www.who.int /tb/features_archive/Spanish_MoscowDeclarationtoEndTB.pdf

8. Stop TB Partnership. Reunión de Alto Nivel de las Naciones Unidas Sobre la Lucha Contra la Tuberculosis. Objetivos y compromisos clave para el 2022. Ginebra: Oficina de Naciones Unidas de Servicios para Proyectos; 2018 [consultado el 24 de marzo del 2020]. Disponible en: http:/ / www.stoptb.org/assets/documents/global /advocacy/unhlm/UNHLM_Commitments_WEB_s.pdf

9. Kritski A, Pretti M, Queiroz F, Calçada A, Rossato D, de Oliveira $\mathrm{M}$, et al. The role of the Brazilian Tuberculosis Research Network in national and international efforts to eliminate tuberculosis. J Bras Pneumol. 2018;44:77-81.
10. Kritski A, Barreira D, Junqueira-Kipnis AP, Moraes MO, Campos MM, Degrave WM, et al. Brazilian response to Global End TB Strategy: The National Tuberculosis Research Agenda. Rev Soc Bras Med Trop. 2016;49:135-45 [consultado el 18 de marzo del 2020]. Disponible en: https://www.scielo.br/scielo.php?script=sci_arttext\& pid=S0037-86822016000100135

11. Yagui M, Jave H, Curisinche M, Gutiérrez C, Romani F. Agenda Nacional de Investigación en Tuberculosis en Perú, 2011-2014. Rev Panam Salud Publica. 2013;33:151-8 [consultado el 8 de abril del 2020]. Disponible en: https:/ / www.scielosp.org/pdf/rpsp/2013.v33n2/151-158

12. República de Perú, Ministerio de Salud. MINSAlanza Red Nacional de Investigación en Tuberculosis para combatir esa enfermedad. Lima: MINSA; 2017 [consultado el 8 de abril del 2020]. Disponible en: https://www.gob.pe/institucion/minsa/noticias/14119-mins a-lanza-red-nacional-de-investigacion-en-tuberculosis-para-com batir-esa-enfermedad

13. Hales S, Lesher A, Ford N, Maher D, Ramsay A, Tran N. Reporting guidelines for implementation and operational research. Bull World Health Organ. 2016;94:58-64 [consultado el 30 de marzo del 2020]. Disponible en: http://dx.doi.org/10.2471/BLT.15.167585

14. República de Colombia, Ministerio de Salud y Protección Social. Resolución 227 de 2020. Bogotá: MSPS; 2020 [consultado el 30 de marzo del 2020]. Disponible en: https://www.minsalud.gov.co/No rmatividad_Nuevo/Resoluci\%C3\%B3n\%20No.\%20227\%20de\%2 02020.pdf

15. Organización Mundial de la Salud. Implementación de la Estrategia Fin de la TB. Aspectos esenciales de la OMS. Ginebra: OMS; 2016 [consultado el 30 de marzo del 2020]. Disponible en: https:/ /www. who.int/tb/publications/2015/end_tb_essential_spanish_web.pdf

16. República de Colombia, Ministerio de Salud y Protección Social. Resolución 1171 de 2019. Bogotá: MSPS; 2019.

17. World Health Organization. A global action framework for TB research. Geneva: WHO; 2015 [consultado el 18 de marzo del 2020] Disponible en: https:/ / www.who.int/tb/publications/global-fram ework-research/en/

18. Pineault R, Daveluy C. La planificación sanitaria. Conceptos, métodos, estratégias. 2. ${ }^{a}$ ed. Barcelona: Masson; 1990.

19. Arbeláez M. Tuberculosis: una ventana de análisis de las políticas públicas en salud. Medicina. 2018;40:31-6. 
20. Marín D, Marín N, del Corral H, López L, Ramírez-Agudelo ME, Rojas CA, et al. PPD-induced monocyte mitochondrial damage is associated with a protective effect to develop tuberculosis in BCG vaccinated individuals: A cohort study. PLoS One. 2017;12:e171930 [consultado el 1 de abril del 2020]. Disponible en: https://doi.org /10.1371/journal.pone.0171930

21. Realpe T, Correa N, Rozo JC, Ferro BE, Gómez V, Zapata E, et al. Population structure among Mycobacterium tuberculosis isolates from pulmonary tuberculosis patients in Colombia. PLoS One. 2014;9:e93848 [consultado el 1 de abril del 2020]. Disponible en: https://doi.org/10.1371/journal.pone.0093848

Manuscrito recibido el 2 de septiembre del 2020. Aceptado para publicación, tras revisión, el 30 de noviembre del 2020.

\section{National Network for Knowledge Management, Research, and Innovation in Tuberculosis in Colombia}

ABSTRACT In 2018, the Colombian Ministry of Health and Social Protection, in collaboration with the National Health Institute and the Pan American Health Organization, convened all actors associated with the National Tuberculosis Prevention and Control Program (PNPCT) and the National Science, Technology, and Innovation System to form a national network to coordinate operations research on tuberculosis (TB) in the country. Seven workshops were held and a consensus was reached to establish the National Network for Knowledge Management, Research, and Innovation in Tuberculosis in Colombia (TB Colombia Network). With more than 180 participants, a central node, and five regional nodes that bring together the country's 37 territorial entities, the TB Colombia Network conducted four days of training in operations research in 2019, defining 65 priority research topics aligned with the strategic lines in the PNPCT: prevention and comprehensive care, with a focus on people affected by TB (41 topics in three modules); and political commitment, social protection, and support systems (24 topics in four modules). The peer comparison method was used for prioritization. The TB Colombia Network remains active and contributes to knowledge management aimed at eliminating TB in Colombia. It has helped make progress in building plans and implementing local operations research projects that generate scientifically validated results to strengthen the PNPCT and improve the care of TB patients.

Keywords Tuberculosis; national health programs; sustainable development; Colombia.

\section{Rede Nacional de Gestão do Conhecimento, Pesquisa e Inovação em tuberculose na Colômbia}

RESUMO

Palavras-chave
O Ministério da Saúde e Previdência Social da Colômbia, em cooperação com o Instituto Nacional de Saúde e a Organização Pan-Americana da Saúde (OPAS), convocou em 2018 todas as partes interessadas vinculadas ao Programa Nacional de Prevenção e Controle da Tuberculose (PNPCT) e ao Sistema Nacional de Ciência, Tecnologia e Inovação para formar uma rede nacional com o objetivo de coordenar a pesquisa operacional em tuberculose no país. Foram promovidos sete seminários e, deste processo, se chegou a um consenso para criar a Rede Nacional de Gestão do Conhecimento, Pesquisa e Inovação em Tuberculose na Colômbia (Rede TB Colômbia). A rede conta com mais de 180 participantes, organizados em um núcleo central e cinco núcleos regionais que congregam 37 entidades territoriais. Em 2019, a Rede TB Colômbia realizou quatro jornadas de capacitação em pesquisa operacional e definiu 65 áreas temáticas prioritárias de pesquisa harmonizadas com as linhas estratégicas estabelecidas pelo PNPCT: prevenção e atenção integral com enfoque nas pessoas com TB (41 áreas temáticas divididas em três módulos) e compromisso político, proteção social e sistemas de apoio (24 áreas temáticas divididas em quatro módulos). A priorização foi definida pelo método de comparação pareada. A Rede TB Colômbia continua ativa contribuindo para a gestão do conhecimento voltado à eliminação da tuberculose no país e possibilitando avançar na formulação de planos e execução de projetos locais de pesquisa operacional com a produção de resultados científicos validados para fortalecer o PNPCT e melhorar a atenção das pessoas com TB.

Tuberculose; programas nacionais de saúde; desenvolvimento sustentável; Colômbia. 